teilweise im weiteren Verlauf der Arbeit noch zurückzukommen sein wird. Für das 17. Jahrhundert erwähnt sie Charles de Saint-Évremont (1613-1703), Jean de La Bruyère (1645-1696), François Fénelon (1651-1715), Bernard le Bovier de Fontenelle (1657-1757) oder Charles Rollin (1661-1741); für das 18. Jahrhundert Voltaire (16941778), Denis Diderot (1713-1784), Claude Adrien Helvétius (1715-1771) oder JeanBaptiste le Rond d'Alembert (1717-1783), denen im 19. Jahrhundert John Stuart Mill (1806-1873) folgt:

[L]'idéal démocratique et individualiste du XVIII est favorable aux femmes ; elles apparais-
sent à la plupart des philosophes comme des êtres humains égaux à ceux du sexe fort. Vol-
taire dénonce l'injustice de leur sort. Diderot considère que leur infériorité a été en grande
partie faite par la société. 'Femmes, je vous plains !' écrit-il. Il pense que : 'Dans toutes les
coutumes la cruauté des lois civiles s'est réunie contre les femmes à la cruauté de la nature.
Elles ont été traitées comme des êtres imbéciles.' Montesquieu estime paradoxalement que
les femmes devraient être subordonnées à l'homme dans la vie du foyer mais que tout les
dispose à une action politique. 'Il est contre la raison et contre la nature que les femmes
soient maîtresses de maison... il ne l'est pas qu'elles gouvernent un empire.' Helvétius
montre que c'est l'absurdité de son éducation qui crée l'infériorité de la femme ; d'Alembert
partage cette opinion [kursiv im Orig.]. (Beauvoir 1976, 185-186)

Insgesamt spricht Beauvoir vom 18. Jahrhundert als einem günstigen Jahrhundert für die Frau, da die meisten ,philosophes' Frauen und Männer als gleichwertige menschliche Wesen anerkennen, auch wenn diese Gleichheit bestimmte Lebensbereiche ausspart.

\title{
4.2 ZUM ZWEI-GESCHLECHTER-MODELL
}

In der Mitte des 18. Jahrhunderts entwickelt sich parallel zum egalitären ein komplementärer Geschlechterdiskurs, der durch die Abkehr von der cartesianischen Denktradition geprägt ist und der es erlaubt, Körper und Geist zusammen und in Abhängigkeit voneinander zu denken (cf. Steinbrügge 1987, 53; ead. 1994, 340). Vermittels des epistemologischen Wandels wird Poulain de la Barres und Feijoos Diskurs über die Gleichheit der Geschlechter von einem anthropologischen Diskurs ${ }^{11}$ überlagert, der die Menschen in zwei ungleiche Teile einteilt, wobei die (Geschlechts-)Körper von Frau und Mann von nun an als komplett verschieden voneinander wahrgenommen werden. Um das neue Modell zu legitimieren, wendet man sich also den Naturwissenschaften zu. Im Unterschied zum Ein-Geschlecht-Modell erkennen diese im Zwei-GeschlechterModell (Laqueur 2003) eine qualitative (anstatt einer graduellen) Differenz zwischen weiblichen und männlichen Geschlechtsmerkmalen, wobei Vulva und Penis als die

11 Damit ist ein Diskurs gemeint, der den anatomischen Körper des Menschen in den Mittelpunkt stellt und von der Anatomie des Menschen ausgeht: „Die erste Definition von Anthropologie im Dictionnaire de Trévoux 1721 lautet: ,Diskurs über den Menschen oder den menschlichen Körper; [und stellt einen] Begriff aus dem Bereich der Anatomie‘ [dar] [kursiv im Orig.]“ (Van Delft 2005a, 167). 
zentralen Unterscheidungsmerkmale fungieren. Diese veränderte äußerliche Wahrnehmung bringt auch eine veränderte Selbstwahrnehmung des Geschlechtskörpers mit sich, der durch die (konstruierte) Existenz zweier biologisch-anatomisch unterschiedlicher Körper künftig anders erlebt und empfunden wird (cf. Maihofer 1995, 92; ead. 1994, 175). Der anthropologische Diskurs setzt zwar ebenfalls bei der Natur ${ }^{12}$ an, allerdings hebt er die Rolle der Frau in ihrer demografischen Verpflichtung hervor, das heißt in der Gattungserhaltung und Brutpflege. ${ }^{13}$ Indem er die Frau auf die ,natürliche‘ Rolle als Ehefrau und Mutter reduziert, wird sie vom sozialen Leben immer mehr ausgegrenzt und in die Privatheit und Häuslichkeit zurückgedrängt (cf. Steinbrügge 1994, 337). Die Natur, das heißt der biologische Geschlechtskörper an sich, wird nun grundlegend anders und als unveränderlich wahrgenommen, sodass in weiterer Folge die Idee einer ,natürlich` begründeten Differenz von zwei Geschlechtern entstehen kann, wobei die Frau der Natur als näherstehend betrachtet und dafür in besonderem Maße geachtet wird.

Diese biologistische Bestimmung von Frau und Mann lässt sich, wie Lieselotte Steinbrügge (1987) zeigt, bereits in der Encyclopédie (1751-1772) ${ }^{14}$ auffinden. In den Artikeln zu HOMME (Hist. nat.), HOMME (Anatomie) und HOMME (Exposition anatomique du corps de l') werden Frau und Mann zunächst, wenn auch nur implizit, als Mensch beziehungsweise menschliches Naturwesen definiert. Erst bei weiterer Lektüre lässt sich eine (ebenfalls implizite) Unterscheidung der Geschlechter unter Ausschluss des weiblichen Geschlechts ablesen, und zwar, wenn es um die Vernunfteigenschaft des Menschen, also des Mannes, geht. Das Menschsein von Frau und Mann wird hier - im Unterschied zu Poulain de la Barre und seinen Nachfolger*innen, die hier nicht einmal mehr erwähnt werden - nicht mehr gleich, sondern unterschiedlich

12 Insgesamt wird der Terminus ,Natur“ zum „Universalbegriff des siècle des lumières [...]. Er ist den französischen aufklärerischen Philosophen sowohl eine wirksame Waffe gegen das irrationale, das heißt religiös geprägte Welt- und Menschenbild als auch gegen den Despotismus und die sozialen Ungerechtigkeiten der feudalistischen Herrschaft. Er ist Ausgangspunkt und Maßstab für die in der Aufklärung entwickelten Utopien und Entwürfe in allen Bereichen des Wissens [...] [kursiv im Orig.]“ (Brink 2008, 355).

13 Michel Foucault (1983, 98-99) beschreibt eine ähnliche Verschiebung in der Betrachtungsweise darüber, was der Mensch, was Leben überhaupt sei. Er konstatiert, dass seit Jahrhunderten Theoretiker*innen davon ausgehen, dass das Leben ein Mechanismus zur Reproduktion und der Sex somit der Grund für alles Leben sei, anstatt davon auszugehen, dass das Leben eine Organisation mit Fortpflanzungsfähigkeit sei. Das, was also als eine Möglichkeit wahrgenommen werden könnte, wurde zur Pflicht erkoren.

14 Auch wenn unter den 140 bekannten Namen der Encyclopédie-Beitragenden nur Namen von Männern aufscheinen (cf. Kafker 1996), so sind doch die Hälfte der rund 74.000 Artikel in der Encyclopédie anonym oder unter einem Pseudonym verfasst worden. Unter den anonymen Autor*innen befinden sich auch Frauen, wie Suzanne-Marie de Vivans Marquise de Jaucourt (1698-1772), die Ehefrau von Pierre-Antoine de Jaucourt, der wiederum der Bruder des Enzyklopädisten Louis de Jaucourt (1704-1780) ist (cf. Haechler 2007). Ebenfalls ist bekannt, dass posthum Texte der Mathematikerin, Physikerin, Philosophin und Übersetzerin Émilie du Châtelet (1706-1749) in die Encyclopédie aufgenommen wurden, ohne sie jedoch als Autorin kenntlich zu machen (cf. Reichenberger 2016, 93-97). 
gedacht: Als Medium einer ,physiokratisch orientierten Gesellschaftstheorie [kursiv im Orig.]" (ibid., 35) verbinden die Encyclopédie-Artikel das Menschsein mit seiner Nützlichkeit, die einer der zentralen Leitbegriffe der Aufklärung ist und sich auf alle Bereiche des kollektiven wie des individuellen Lebens erstreckt. ${ }^{15}$

Als Leitbild des innerhalb der bürgerlichen Gesellschaft nützlichen Mannes gilt der Honnête homme, der ,sich als Mensch durch gesellschaftlich nützliche Tätigkeit“ (Steinbrügge 1987, 34) realisiert. ${ }^{16}$ In der Encyclopédie ist diese nützliche, i. e. produktive Tätigkeit ,ausschließlich als ,Männergesellschaft' beschrieben. Die Suche nach der honnête femme, dem weiblichen Gegenpart zum honnête homme bleibt vergeblich [kursiv im Orig.]“ (ibid., 35). Während nun der menschliche Wert des Mannes in seiner in der öffentlichen Sphäre stattfindenden handwerklichen Produktivität (Arbeit) bestimmt wird, wird der Wert der Frau auf ihre biologische (Re-)Produktivität (Fruchtbarkeit) und die daraus resultierenden Verpflichtungen in der privaten Sphäre beschränkt - und das obwohl Frauen in gleichem Maße wie Männer am ökonomischen Leben der Zeit teilhaben. Die Nützlichkeit von Frau und Mann innerhalb der Gesellschaft wird als komplementär erachtet, was sich auch in für Frauen und Männer unterschiedlichen Tugendkodizes widerspiegelt, auf die weiter unten eingegangen wird. Allein schon an den Artikelarten und der -anzahl innerhalb der Encyclopédie lässt sich ablesen, dass die biologische Natur der Frau über ihrer gesellschaftlichen Arbeit steht. Ferner verbirgt sich hinter den Stichworten ,Gebärmutter', ,Geburt', ,Brüste‘, ,Amme“ oder ,Menstruation' das gesammelte Wissen der Zeit über den weiblichen Körper, wobei der Fokus stets auf dessen Gebär- und Stillfähigkeit liegt. Bei allen Zuweisungen in die private Sphäre wird der Frau in der Encyclopédie jedoch noch nicht die Fähigkeit zum vernünftigen Denken abgesprochen. Dies geschieht erst in den 1770er-Jahren durch den Historiker Antoine-Léonard Thomas und den Philosophen und Mediziner Pierre Roussel (cf. ibid., 32-43), auf die im Anschluss an ihren Wegbereiter Rousseau eingegangen wird.

15 Die gesellschafts- und wirtschaftstheoretische Schule der Physiokraten, die im Frankreich der zweiten Hälfte des 18. Jahrhunderts entsteht, betont, dass eine optimale Ausnutzung des landwirtschaftlichen Territoriums zu Reichtum, Stabilität und Ansehen eines Volkes führe. Als Voraussetzung für den ökonomischen Fortschritt gilt den Physiokraten eine steigende Bevölkerungszahl, die wiederum auf der biologischen Reproduktion beruhe (cf. Schaufler 2002, 125; Steinbrügge 1987, 12; Höfer/Keilhauer 1996, 45). Die menschliche Bestimmung der Frau wird folglich ,auf die ,nützlichen“ Potentiale ihres Körpers, auf ihre Reproduktionsfähigkeit und damit auf ihre Geschlechtlichkeit [beschränkt]“ (Schaufler 2002, 127). Sexualität diene lediglich ,der Fortpflanzung des Volkes [...]. In dieser physiokratischen Anschauung erscheint die ,zweckfreie‘ Lust als Gefahr für den Ehestand und als Vergeudung von Kräften und Säften“ (ibid., 160).

16 Während das Honnête-homme-Ideal im 17. Jahrhundert für ein adeliges Standesideal mit den Komponenten einer hohen Geburt, geistreicher Höflichkeit und Salonmanieren steht, gewinnt es ab Ende des 17. Jahrhunderts eine allgemein universelle Vorbildlichkeit. Im 18. Jahrhundert wird das Persönlichkeitsideal immer mehr mit bürgerlichen, moralisch-ethischen Qualitäten verbunden, die auf Tugend, Mitmenschlichkeit und Aufrichtigkeit fußen. Zur Entwicklung und zum Bedeutungswandel des Honnêteté-Begriffs im 17. und im 18. Jahrhundert siehe Anette Höfer und Rolf Reichardt (1986). 
Der entscheidende Propagandist des komplementären Geschlechterverständnisses ist Jean-Jacques Rousseau (1712-1778), auch wenn er nicht von einer biologistischen Ungleichheit der Geschlechter, sondern von einer instrumentell-funktionalistischen Geschlechterdifferenz ausgeht. Der Kritiker und Gegner der Aufklärungsbewegung (und des Absolutismus) (cf. Schneiders 1997, 73) leitet die Ungleichheit zwischen Frau und Mann ,aus einer (vermeintlichen) sozial-evolutionären Entwicklung der Menschen ab, d. h.[,] sie sei für Rousseau zwar ,natürlich', gleichzeitig sei sie aber nicht ,natürlich', da sie [die Ungleichheit] im Naturzustand noch nicht existiert" (Klapeer 2014, 127). Die Entwicklung der sozialen Geschlechterdifferenz begründet er im Discours sur l'origine et les fondements de l'inégalité parmi les hommes (1754) mit der zunehmenden familialen Vergesellschaftung, der sukzessiven sozialen Differenzierung und der Sprach- und Eigentumsbildung, womit ebenfalls die Ausbildung einer Moralität einhergeht. Mit der gesellschaftlichen Evolution treten Frau und Mann demnach in eine moralische Beziehung zueinander, die - aufgrund der als produktiver angesehenen Arbeit des Mannes - der Mann dominiere (cf. Klapeer 2014, 130-131). Im 5. Buch von Émile ou de l'Éducation (1762) schildert Rousseau das Geschlechterverhältnis zwischen dem jungen Mann Émile und Sophie, die seine Ehefrau werden soll, folgendermaßen:

Dans l'union des sexes chacun concourt également à l'objet commun, mais non pas de la même manière. De cette diversité naît la première différence assignable entre les rapports moraux de l'un et de l'autre. L'un doit être actif et fort, l'autre passif et faible : il faut nécessairement que l'un veuille et puisse, il suffit que l'autre résiste peu.

Ce principe établi, il s'ensuit que la femme est faite spécialement pour plaire à l'homme. Si l'homme doit lui plaire à son tour, c'est d'une nécessité moins directe : son mérite est dans sa puissance ; il plaît par cela seul qu'il est fort. Ce n'est pas ici la loi de l'amour, j'en conviens ; mais c'est celle de la nature, antérieure à l'amour même.

Si la femme est faite pour plaire et pour être subjuguée, elle doit se rendre agréable à l'homme au lieu de le provoquer; sa violence à elle est dans ses charmes; [...]. (Rousseau 1762, Livre V, 6)

Die Bildung von Sophie fällt dementsprechend anders aus als die von Émile. Während er zu einem selbständigen, autonomen und rationalen Bürger herangezogen wird, wird Sophie gelehrt, ihre Pflichten gegenüber dem Ehemann zu lieben und diese verständig und mit Freude auszuführen. Sie muss nicht belesen, sondern lediglich anmutig sein (cf. Lerner 1993, 211). ${ }^{17}$ Mit Rousseau wird die Unterordnung der Frau unter den Mann demnach zu einer Selbstverständlichkeit, die diese ,,als einen ,natürlichen“ Akt

17 Wolfgang Martens (1968) hält fest, dass die deutschsprachigen Moralischen Wochenschriften Rousseaus ,Ideal der aimable ignorante, des anmutig-unwissenden, empfindsamen weiblichen Geschöpfs“ (532) nicht verfolgen, sondern sich dafür aussprechen, dass Frauen im Umgang mit Literatur geschult und gebildet werden. „Lesender Umgang mit den nützlichen und schönen Wissenschaften klärt auf, bessert Verstand und Willen, befördert die Tugend, dies wird dem Publikum der Wochenschriften immer wieder bedeutet. Aber auch über die allgemein versittlichenden und bildenden Wirkungen hinaus sind glückliche Effekte zu erwarten. Man verweist auf greifbaren Nutzen“ (ibid.). 
freiwilliger Hingabe begreifen“ (Höfer/Keilhauer 1996, 48) und lieben lernt. Die Umdeutung des Liebesbegriffs unterstützt diese Entwicklung. Rousseau spricht sich für eine (romantische) Liebesheirat zwischen Frau und Mann aus, womit er ,sowohl zu einer Verinnerlichung der Zweierbeziehung als auch zur Ausprägung der Idee der Familie als Hort privater Intimität“ (ibid., 47-48) tendiert. Somit überhöht Rousseau auch die Mutterschaft zu einem ,natürlichen', freiwilligen Liebesbeweis der Frau gegenüber ihrem Ehemann und ist nun nicht mehr als Pflicht zum Erhalt der Institution der Familie anzusehen. Der Wirkungsbereich der Frau beschränkt sich schließlich auf die private Sphäre, in der sie - obwohl sie aus dem öffentlichen Leben ausgeschlossen wird eine politische Rolle erhält. In der privaten Sphäre des Hauses soll sich die Frau der Erziehung der Kinder, also der künftigen Bürger*innen, widmen und somit am Aufbau und Erhalt der Republik sowie an der Erneuerung des Gemeinschaftssinns mitwirken (cf. Pečar/Tricoire 2015, 158-159).

Ein weiterer Propagandist des komplementären, hierarchischen Geschlechterverständnisses ist der Historiker Antoine-Léonard Thomas (1732-1785). In seinem Essai sur le caractère, les mours et l'esprit des femmes dans les différens siècles (1772) liefert er zunächst eine Zusammenfassung der Querelles des femmes seit Jahrhundertmitte - dieser erste Teil erscheint durch seine Auflistung der Leistungen von Frauen als traditionelle Frauenapologie - und schließt im nächsten Teil an die moralphilosophischen Überlegungen über die ,Natur der Frau' von Rousseau an. ${ }^{18}$ Seine Argumentation wandelt sich also von einem ,rationalistisch begründeten Egalitätspostulat zu einer sensualistisch geprägten Geschlechtertheorie“ (Pabst 2007, 52), in der Thomas eine natürliche Geschlechterdifferenz annimmt. Aus der unterschiedlichen Anatomie der Geschlechter leitet er ab, dass die Frau weniger intellektuelle Fähigkeiten als der Mann besitze. Zudem postuliert Thomas einen Einfluss der Sinnesorgane auf die menschliche Erkenntnisfähigkeit und eine größere Empfindsamkeit (sensibilité) der Frau, ${ }^{19}$ woraus er weiter ableitet, dass „Frauen zu bestimmten kognitiven Leistungen

18 In Spanien findet die Kategorie der Natur in den Texten der Querelles des sexes von Benito Jerónimo Feijoo y Montenegro (1676-1764) und Gaspar Melchor de Jovellanos (1744-1811) weniger Beachtung als in Frankreich, was mit der religiösen Fundierung der spanischen Aufklärung in Zusammenhang stehen dürfte, die die Vernunft nicht als natur-, sondern als gottgegeben annimmt. Stärker als Natur und Vernunft wird hier die Kategorie der Nützlichkeit in den Vordergrund gerückt (cf. Brink 2008, 359-360; Kilian 2002, 187).

19 Ideengeschichtlich entspringt die Ethik des Gefühls durch die in England und Schottland diskutierte Theorie des ,moral sense' sowie die in Frankreich stattfindende Diskussion um die ,sensibilité‘ und den deutschen Pietismus. Während in Frankreich zu Beginn der Empfindsamkeitsdiskussion als ihr Hauptmerkmal das Gefühl der Mitleidsfähigkeit und der Empfänglichkeit für ein Gefühl der Liebe, das die physische Anziehungskraft übersteigt, verstanden wird, bringt man es ab 1704 in die Nähe einer natürlichen Moral - einem angeborenen Gefühl. Im 17. Jahrhundert hat „die sensibilité keine spezifische soziale oder geschlechtliche Zuordnung [kursiv im Orig.]“ (Sauder 1992, 115). Durch Julie ou La Nouvelle Heloise (1761) - Archetypus des sentimentalen Romans - von Jean-Jacques Rousseau wird die ,sensibilité' schließlich zur dominierenden Modeerscheinung der Zeit (cf. Sauder 1992; id. 1997). 
nicht in der Lage seien“ (Steinbrügge 1987, 52; cf. Brink 2008, 357-359; Honegger 1992, 141; Steinbrügge 1987, 47-52).

Es ist schließlich der französische Philosoph und Mediziner Pierre Roussel (17421802), der im Zuge der anthropologischen Wesensbestimmung beide Geschlechtskörper vergleichend in den Blick nimmt und die menschliche Anatomie mit der Philosophie (= Moral) verknüpft, wobei die Anatomie in weiterer Folge zur „Grundlagenwissenschaft der entstehenden Wissenschaften vom Menschen wird" (Maihofer 1995, 24). In seinem Système physique et moral de la femme (1775) entwirft er eine ,weibliche Sonderanthropologie', ${ }^{20}$ die den Unterschied von Frau und Mann über die Geschlechtsorgane hinaus unter Einbezug des Knochenbaus, der Nerven, Bänder, Gefäße, Muskeln und Organe ersinnt. Aufgrund der kleineren, weicheren und schwächeren Verfasstheit der weiblichen Organe nimmt Roussel an, dass die Empfindungen, die Vorstellungskraft sowie ein zärtliches Grundgefühl beim weiblichen Geschlecht überwiegen und die Empfindsamkeit der Frau derjenigen des Mannes in physischer wie in moralischer Hinsicht weit überlegen sei. Daraus folgert er, Frauen und Männer würden anders denken, fühlen und urteilen; ferner sei die Frau infolge ihrer Physiognomie und Anatomie nicht zur intellektuellen Arbeit und zur Beschäftigung mit den abstrakten Wissenschaften fähig (cf. Honegger 1992, 144-148; Steinbrügge 1994, 339):

Il n'est pas douteux que cette foiblesse, que nous avons dit caractériser les organes de la femme, ne lui interdise les efforts de cette contention d'esprit qui est nécessaire à l'étude des sciences abstraites, même pour s'y égarer ; \& que son imagination trop mobile, \& peu capable de garder une assiete permanente, ne la rende peu propre aux arts qui dépendent de cette faculté de l'ame : mais aussi c'est de cette foiblesse que naissent ces sentiments doux $\&$ affectueux qui constituent le principal caractère de la femme ; [...]. (Roussel 1775, 31-32)

Aus Roussels Überlegungen wird von späteren ,médecins-philosophes` abgeleitet, dass die geistige Betätigung der Frau die Ermattung ihrer reproduktiven Organe zur Folge hätte (cf. Honegger 1992, 151-152, 163). Ausgehend von dieser physiologischanatomischen Differenz der Geschlechter werden wiederum unterschiedliche Geschlechterrollen und -identitäten induziert (cf. Maihofer 1995, 24-25). Die soziale Rolle der Frau sei die der Ehefrau, Hausfrau und Mutter, deren einzige, sozusagen biologisch-natürliche Aufgabe es sei, Kinder zu gebären und großzuziehen, während

In Frankreich entwickelt sich vor der englischen Empfindsamkeitsrezeption eine eigenständige Diskussion über die ,sensibilité, wobei „[d]ie indirekte Vermittlung der englischen philosophischen Moral der Empfindsamkeit durch die Moralischen Wochenschriften [...] nicht hoch genug zu veranschlagen [ist]“ (Sauder 1992, 112).

20 Claudia Honegger (2011) sieht ,,[m]it dem Werk von Pierre Roussel [...] in den siebziger Jahren des 18. Jahrhunderts die Konstituierung der weiblichen Sonderanthropologie bereits weitgehend abgeschlossen: Die Frau ist ein Wesen für sich, mit einer eigenen Körperlichkeit, eigenen Krankheiten, eigenen Sitten, eigener Moral und eigenen kognitiven Fähigkeiten. Die Artikel über die Frau in der Enzyklopädie markieren die Übergangszeit: Die ältere Anatomie hatte die Frau als körperlich fast gleich angesehen, sie als einen homme manqué betrachtet, worüber der Artikel Femme (Anthropologie) [...] im 6. Band der Encyclopédie (von 1756) noch Auskunft gibt [kursiv im Orig.]“ (Honegger 2011, 110-111). 
der Mann als untauglich für die Kinderaufzucht gilt (cf. Honegger 1992, 161). ${ }^{21}$ Gleichzeitig erfährt die Frau durch die Beschränkung auf diese ,natürliche' Rolle und die damit verbundene Zurückdrängung in Privatheit und Häuslichkeit - wie bereits erwähnt - eine immer stärkere Ausgrenzung aus dem sozialen Leben (cf. Steinbrügge 1994, 337). Der Weiterbestand der Gattung wird somit zur moralischen Verpflichtung der Frau stilisiert. Die Frau wird zur moralischen Instanz, zum sogenannten ,moralischen Geschlecht ${ }^{\circ 22}$ der bürgerlichen Gesellschaft erkoren, während der Mann in der öffentlichen Sphäre ohne Moral auskommt und sozusagen ,morallos' ${ }^{6}$, nur mit Bedacht auf ökonomische Vorteile, agieren kann (cf. ead. 1987, 14). Steinbrügge nennt das Beispiel aus der Encyclopédie zur FEMME (Morale), in dem die von Frauen geführten Salons als unmoralische Orte dargestellt werden und das weibliche Geschlecht bereits in anthropologischer, sozialer und moralischer Hinsicht verortet wird:

[...] anthropologisch als ,Regentin' des Reproduktionsbereichs der Gattung Mensch; sozial als (bürgerliche) Hausfrau und Mutter; moralisch als zurückgezogen lebende, keusche Person, deren Bestimmung (zu lieben) sich ausschließlich im familiären Bereich manifestiert. (Steinbrügge 1987, 42)

21 Das Konzept der ,Mutterrolle', wie es heute in westlichen Ländern geläufig ist, sowie die modernen Begriffe der ,Familie‘ oder der ,Hausarbeit‘ entstehen ,erst mit dem Aufkommen der bürgerlichen Familie im Europa des 17./18. Jahrhunderts“ (Bührmann et al. 2014, 34). Die Frauen der Unterschichten arbeiten gemeinsam mit ihren Ehemännern in der Werkstatt, auf den Feldern oder auf der Straße, während die Kinderstube als Arbeitsplatz nicht existiert. Auch in den bürgerlichen Schichten gibt es bis zur Mitte des 18. Jahrhunderts ,[k]eine von der Erwachsenenwelt abgetrennte Sphäre des Kindes“ (ibid., 36). Kinder werden auf das Land zu bezahlten Ammen und bereits mit 8, 9 oder 10 Jahren in eine Lehre geschickt. Bürgerliche Familien lassen eine Amme ins Haus kommen. Abgesehen von „der Aufbewahrung des Säuglings durch die Mutter oder die bezahlte Amme, die etwa zwei Jahre still[...]en, [gibt es] keine Sozialisation, die allein Aufgabe der Frauen als Mütter [...] wäre: keine bewußten Erziehpraktiken, kein reflektiertes ,kindgemäßes` Verhalten, kein ,mütterliches Eingehen' auf das Kleine, kein Reinlichkeitstraining“ (ibid., 37). Auch ,Kinderspiele“ sind inexistent, denn Kinder werden de facto als „kleine Erwachsene“ (ibid.) angesehen. Diese kulturellen Lebensformen ändern sich erst im Zuge der Aufklärung, und zwar „zunächst in den bürgerlichen Schichten, die als ,Pioniere‘ der modernen Kleinfamilie gelten können. In dieser Zeit setzt[...] eine massive bürgerliche Reformbewegung gegen die alten Arten der Kinderaufzucht ein, und in dem Maße, wie sie sich durchsetzt[...], entst[eht] die ,Mutterrolle‘. Es entst[eht] die Kindheit und mit ihr die Mehr-Arbeit der Frauen in der Kinderstube“ (ibid.).

22 Das Bild der Frau als das ,moralische Geschlecht', das vor allem auf den Entwürfen JeanJacques Rousseaus basiert, wird innerhalb der späteren Querelles des sexes zum Leitbild vieler Debatten, die sich nunmehr um das Gefühl und nicht mehr um den Verstand der Frau drehen: Die Debatten verlagern ,sich in der zweiten Hälfte des 18. Jahrhunderts auf die Ebene des Streits um die moralische Kompetenz der beiden Geschlechter [...]. Die Existenz von Geschlechtscharakteren wird grundsätzlich nicht mehr in Zweifel gezogen. Die Frage nach dem Anteil der Frau am Fortschritt des menschlichen Geistes ist negativ entschieden. Zur Disposition steht nur noch die gesellschaftliche Reichweite ihrer Gefühle“ (Steinbrügge 1987, 118; cf. ibid., 67-96). 
Diese während des gesamten Jahrhunderts verhandelte Neubestimmung der Rollen und Funktionen von Frau und Mann für das Gemeinwohl geht nicht nur mit einer Verschiebung der Auffassung der menschlichen Natur einher, sondern auch mit der Verschiebung der Auffassung von Tugendhaftigkeit (vertu), die zu einem (weiteren) zentralen (geschlechtsspezifischen) Leitbegriff der Aufklärung wird. ${ }^{23}$ Ursprünglich ist der Begriff der Tugend (lateinisch ,virtus“) mit der „Gesamtheit der verdienstvollen Eigenschaften und Qualitäten“ (Pabst 2007, 25) von Männern (lateinisch ,vir`) konnotiert (z. B. Tapferkeit, körperliche Kraft, Mut) ${ }^{24}$ und erlangt ab dem Ende des 17. Jahrhunderts eine Feminisierung. Durch das sich nach und nach durchsetzende binäre und komplementäre Geschlechterverständnis, das Frauen und Männern gegensätzliche, anthropologisch invariante Charaktere und Eigenschaften zuschreibt, wird der ,an männlichen Qualitäten ausgerichtete[...] und für beide Geschlechter einheitlich definierte[...] Tugendbegriff [zusehends] problematisch [...]“" (ibid., 28), sodass der Begriff mit neuen geschlechtsspezifischen Eigenschaften und Verhaltensnormen aufgeladen wird. Im Zuge dieses Bedeutungswandels wird die ,vertu' allerdings nicht nur feminisiert, sondern - und mit Blick auf den anthropologischen Diskurs der Zeit wenig verwunderlich - gleichzeitig auch emotionalisiert, sodass die ,weibliche Tugend“ immer mehr auf dem Gefühl begründet und zunehmend in der privaten Sphäre situiert wird (cf. ibid., 30). Dadurch wird es ermöglicht, die bestehenden hierarchischen (und religiös gerechtfertigten) Geschlechterverhältnisse mit anderen Argumenten weiterzuführen. Im Kontext des Übergangs vom Ein-Geschlecht- zum Zwei-Geschlechter-Modell werden also nicht nur ,geschlechtsspezifisch differenzierte Verhaltensnormen“ (ibid., 28) an den Tugendbegriff gebunden, sondern „die Aufspaltung des bis dato männlich konzipierten Bedeutungsfelds von vertu in nach Geschlechtern getrennte Sinnzuschreibungen und die Konstruktion einer spezifisch weiblichen Tugend [kursiv im Orig.]“ (ibid.) trägt gleichzeitig ,,mit bei zu Erzeugung, Verbreitung und Stabilisierung der Lehre von den polarisierten Geschlechtercharakteren“ (ibid.). ${ }^{25}$

Ein Blick in die Encyclopédie zum Eintrag zur VERTU (Politique) zeigt, dass der Tugendbegriff zur Mitte des Jahrhunderts noch sehr allgemein und vor allem geschlechtsunspezifisch angenommen wird:

23 Zur gleichzeitigen Ausbildung des dualistischen Geschlechtermodells und dem Bedeutungswandel von ,vertu', siehe die Monografie Die Erfindung der weiblichen Tugend von Esther Suzanne Pabst (2007), in der sie dem französischen Tugenddiskurs in Briefromanen aus der zweiten Hälfte des 18. Jahrhunderts nachgeht.

24 Durch die fortschreitende Christianisierung im Mittelalter erhält der Tugend-Begriff eine moralische Erweiterung im Sinne einer moralischen Stärke des Mannes. Dieser männlichen Tugend wird „eine spezifisch weibliche Komponente im semantisch ganz begrenzten Sinne von chasteté (Keuschheit) hinzugefügt [kursiv im Orig.]“ (Pabst 2007, 26).

$25 \mathrm{Im}$ Laufe des 18. und 19. Jahrhunderts kommt es, wie Karin Hausen (1976) gezeigt hat, zu einer Polarisierung der ,Geschlechtscharaktere‘. Ausgehend vom biologischen Geschlecht werden Frauen Charaktereigenschaften im Zusammenhang mit Emotionalität zugeschrieben, und Männern Charaktereigenschaften, die mit Rationalität in Verbindung gebracht werden. Daraus ableitend konzipiert man geschlechtsspezifisch geteilte Arbeit sowie die Dissoziation von Erwerbsleben (öffentliche Sphäre) und Familienleben (private Sphäre). 
VERTU, (Ord. encyclop. Mor. Polit.) il est plus sûr de connoître la vertu par sentiment, [...]. Le sentiment ne se connoit que par le sentiment. [...]

Le mot de vertu est un mot abstrait, qui n'offre pas d'abord à ceux qui l'entendent, une idée également précise \& déterminée ; il désigne en général tous les devoirs de l'homme, tout ce qui est du ressort de la morale ; un sens si vague laisse beaucoup d'arbitraire dans les jugemens ; [...]; il est vrai qu'en général les hommes s'accorderoient assez sur ce qui mérite le nom de vice ou de vertu, si les bornes qui les séparent étoient toujours bien distinctes ; mais le contraire arrive souvent [kursiv im Orig.]. (Diderot/D'Alembert 2016, 17:176)

Für die ,philosophes‘ der Aufklärung entspringt die ,vertu‘ zum einen aus einem Gefühl, ist also ,von Natur aus“ in jedem Menschen vorhanden (,Le sentiment ne se connoit que par le sentiment“" [Diderot/D’Alembert 2016, 17:176]), und zum anderen aus dem moralischen Bereich heraus (,tout ce qui est du ressort de la morale“ [ibid.]). Die ,vertu' wird somit als ein angeborenes moralisches Gefühl (morale de sentiment) konzipiert, das im Wesentlichen darauf ausgerichtet ist, den Mitmenschen Gutes zu tun beziehungsweise ihnen nützlich zu sein. Was dieses Gute und Nützliche ist, hängt vor allem von der Intention ab, mit der ein Mensch handelt, denn Gnade/Milde (clémence) walten zu lassen, könne zum Beispiel zuweilen als Tugend, zuweilen als Laster gewertet werden. Die Beweggründe für das Handeln der Menschen könne man über die Konsultation des Geistes (esprit), des Verstandes (raison), des Gewissens (conscience) beziehungsweise des moralischen Instinkts (instinct moral) eruieren:

[S]ur-tout il faut juger des actions par les motifs, [...]; plus l'intention est pure, plus la vertu est réelle. Eclairez donc votre esprit, écoutez votre raison, livrez-vous à votre conscience, à cet instinct moral si sûr \& si fidelle, \& vous distinguerez bientôt la vertu, car elle n'est qu'une grande idée, ou plutôt qu'un grand sentiment [kursiv im Orig.]. (Diderot/D'Alembert 2016, $17: 176)$

Tugend und Gefühl werden hier auf eine Stufe gehoben, wobei die Vernunft als das geeignete Mittel angesehen wird, um die Tugend zu erkennen. Daraus leitet sich ab, „dass wahre Tugend sentiment ebenso wie raison fordert, dass beide zusammenwirken müssen, damit das Ziel der Tugendhaftigkeit erreicht werden kann [kursiv im Orig.]“ (Hillesheim 2013, 52). Wer also den Verstand einschaltet, kann selbst darüber befinden, ob eine Handlung oder ein Charakter tugend- oder lasterhaft ist, wobei effektiv der Verzicht auf das Laster propagiert wird: Wer tugendhaft ist, wird von der Gesellschaft (oder bei Voltaire und Rousseau von einem ,Être suprême') belohnt (i. e. wertgeschätzt), wer lasterhaft ist, wird von der Gesellschaft (und ihren Gesetzen) sanktioniert (cf. Domenech 1997, 1085-1088). Dieses Prinzip ist laut Encyclopédie allen Menschen - Frauen wie Männern - als ,vérité de sentiment' (Wahrheit des Gefühls), also ,von Natur aus' gemeinsam: „que la vertu soit aimable \& digne de récompense, que le vice soit odieux \& digne de punition, c'est une vérité de sentiment à laquelle tout homme est nécessité de souscrire [kursiv im Orig.]“" (Diderot/D’Alembert 2016, 17:177). Außerdem wird Tugendhaftigkeit mit Glück (bonheur) gleichgesetzt, denn nur wer ein tugendhaftes Leben führt, kann zu seinem eigenen Glück und zum Glück 
der Gemeinschaft beitragen. Tugendhaftigkeit wird also als Mittel angesehen, um das individuelle und kollektive Ziel des Glücks zu erreichen. ${ }^{26}$

Die Tugenden werden demnach als gesellschaftlich nützliche Qualitäten einer Person angesehen. Ihre Nützlichkeit leitet sich von einer auf Vernunft basierenden weltlichen Morallehre ab, die in der Aufklärung neben der christlichen Morallehre konzipiert wird und sich auf die diesseitigen Bereiche des Lebens und des sittlichen Miteinanders bezieht. Tugend- wie Lasterhaftigkeit werden von den ,philosophes' als ,natürliche“ Eigenschaften von Menschen aufgefasst. Während Tugenden nun auf den allgemeinen Nutzen ausgerichtet sind und der Gesellschaft zugutekommen sollen, stellen Laster laut Encyclopédie-Eintrag zu VICE (Droit naturel, Morale, \&c.) verwerfliche moralische Eigenschaften dar, die in der falschen Bemessung des Guten liegen: „[L]e fondement du vice consiste dans les fausses mesures du bien [kursiv im Orig.] (Diderot/D'Alembert 2016, 17:235). Fast alle sogenannten sozialen Tugenden (vertus sociales) entspringen der natürlichen, instinktiven Fähigkeit der Menschen, mit Anderen mitleiden (pitié), das heißt mitfühlen zu können, indem sie sich in andere Menschen hineinversetzen und sich mit ihnen identifizieren:

Il est prouvé que la pitié est naturelle à l'homme, $[\ldots]$; or ce sentiment seul est la source de presque toutes les vertus sociales, puisqu'il n'est autre chose qu'une identification de nousmêmes avec nos semblables, \& que la vertu consiste sur-tout à réprimer le bas intérêt \& à se mettre à la place des autres [kursiv im Orig.]. (Diderot/D'Alembert 2016, 17:178)

Anders ausgedrückt, ihre Einhaltung ist nützlich und notwendig für den Einzelnen wie für das Leben im Kollektiv: „la vertu est également essentielle aux états \& aux particuliers [kursiv im Orig.]“ (ibid. 17:178).

Wie die Tugenden konkret aussehen, bleibt der Encyclopédie-Artikel zur ,vertu“ schuldig. ${ }^{27}$ Nur ein paar fassbare Hinweise zu den für alle Menschen geltenden sozialen Tugenden treten im Laufe des Eintrags hervor. ${ }^{28}$ Einzig und allein die Keuschheit

26 Das gesamte 18. Jahrhundert über wird fast zwanghaft nach dem Glück gesucht, das schließlich in die Erklärung der Menschen- und Bürgerrechte von 1793 Eingang findet. Dies lässt sich dadurch erklären, dass im Aufklärungszeitalter die Suche nach dem irdischen Glück als letztliche Bestimmung des Menschen gegen den Glauben an das jenseitige Glück eingetauscht wird. Wie sich in den untersuchten spanischen Wochenschriften zeigen wird, geht man immer mehr davon aus, dass vor allem die Bildung den Menschen dabei unterstützen kann, dieses Glück zu finden (cf. Labrador/Pablos 1989, 74-75).

Siehe Robert Mauzi (1969) L'idée du bonheur dans la littérature et la pensée françaises au XVIIIe siècle; dezidiert zur Konzeption von ,happiness“ im Spectator siehe Norton (2015) „The Spectator, Aesthetic Experience and the Modern Idea of Happiness“.

27 Ebensowenig findet sich eine konkrete Bezeichnung der Laster im Artikel zum VICE (Droit naturel, Morale, \&c.).

28 Der Artikel nennt die folgenden Tugenden: „[1’]amour pour la modération, la tempérance, la simplicité, la frugalité", „[être] tempérant“, „éviter[...] les excès qui pourroient mi [sic] nuire“, ,n'offenser[...] point les autres par la crainte des réprésailles“, ,[être] poli[...]“, ,,modérer le[...]s desirs“; zu den konkreteren Lastern werden ,[1]'intérêt, les préjugés, les passions" gezählt. 
(chasteté) wird im weiteren Eintrag zur VERTU (Langue françoise) der Frau allein zugeordnet. Dass es jedoch einen für Männer und Frauen unterschiedlichen Tugendkodex in der Encyclopédie gibt, hat Esther Suzanne Pabst (2007, 32-47) anhand der Betrachtung der Verweiseinträge gezeigt, die sie über den Eintrag DEVOIR (Droit nat. Relig. nat. Morale) zu den Einträgen zur FEMME (Morale) und zum HOMME (Morale) führen. Indem hier die gesellschaftlichen Pflichten (devoirs) des Menschen entworfen werden, werden auch gesonderte weibliche und männliche Tugendattribute genannt und näher ausgeführt. Auf der einen Seite steht die ,weibliche', auf dem Gefühl basierende ,vertu' der Frau, die mit ihren ,natürlichen` Tugenden wie Barmherzigkeit, Liebe, Zärtlichkeit, Güte und Sanftmut ihren gesellschaftlichen Pflichten als Ehefrau, Hausfrau und Mutter nachkommen kann. Auf der anderen Seite steht die auf der Vernunft basierende ,vertu' des Mannes, der seine ,natürliche' Vernunftbegabung zum wissenschaftlichen Fortschritt und zur Beherrschung der Natur einzusetzen hat. Unter Berücksichtigung der Verweiseinträge stellt sich auch die Bestimmung der Tugend im VERTU-Artikel der Encyclopédie als geschlechtsspezifisch heraus und unterscheidet sich wie folgt: ,die auf dem intuitiv-instinkthaften Gefühl gründende Tugend des vulgaire versus vernunftgeprägte Tugend des philosophe [kursiv im Orig.]“ (ibid., 44). Während die auf dem moralischen Instinkt fußende ,vertu' mit dem Weiblichen assoziiert wird, wird die auf vernünftige Überlegungen und Einsichtsfähigkeit fußende ,vertu“ mit dem Männlichen assoziiert. Vermittels der „Komponente der rational gesteuerten Selbstdisziplinierung im Namen des übergeordneten Gemeinwohls“ (ibid., 45) steht die ,männliche vertu‘ zudem hierarchisch über „,dem natürlich-ungezwungenen Hang zu Tugend“" (ibid.) der Frau.

Die Annahme einer natürlichen Differenz zwischen den Geschlechtern, die andeutungsweise bereits in der Encyclopédie vorhanden ist, wird bei Antoine-Léonard Thomas im zweiten Teil der Essais weiter ausgebaut. In seinem Differenzdiskurs, den er von einer divergenten organischen Beschaffenheit von Frau und Mann ableitet, geht er nicht nur von andersartigen intellektuellen Fähigkeiten, sondern auch von unterschiedlichen Tugenden bei Frau und Mann aus: Die auf dem Gefühl basierenden Tugenden (vertus) der Frau kontrastiert er mit den auf dem Verstand basierenden intellektuellen Fähigkeiten (talents) des Mannes. Die grundsätzlich bei der Frau angesiedelten Tugenden unterteilt Thomas weiter in die ,vertus religieuses', die ,vertus domestiques' und die ,vertus sociales', durch deren Erfüllung die Frau im privat-häuslichen Bereich als Ehefrau, Hausfrau und Mutter den für sie prädestinierten sozialen Beitrag erfüllen kann. Im Zusammenspiel der weiblichen altruistischen Gefühlstugend mit der männlichen egoistischen Verstandeskraft sieht Thomas schließlich das Wohl der Gesellschaft gesichert, wobei den weiblichen Tugenden eine größere Bedeutung zukomme, denn auf diesen ,gründet Thomas' Argumentation zufolge das moralische Funktionieren und damit die Stabilität der gesamten Gesellschaft. Denn nur in der Erfüllung ihrer moralischen Überlegenheit qua Weiblichkeit könnten die Frauen die Defizite der Männer in diesen Bereichen kompensieren und vorbildhaft wirken“ (Pabst 2007, 68).

Auch für Pierre Roussel resultieren aus der ,natürlich`schwächeren organischen Disposition ,typisch' weibliche Tugenden, wie Sanftmut, Anteilnahme oder Mitleid (pitié). Schwäche (faiblesse) und Empfindsamkeit (sensibilité) werden als „Geschenke der Natur“ (Honegger 1992, 150) dargestellt, denn gäbe es nur rationale (männliche) Wesen, wäre die Menschheit längst ausgestorben: „Cette organisation étoit sans doute 
nécessaire dans le sexe à qui la nature devoit confier le dépot de l'espece humaine encore faible \& impuissante. Celle-ci eût mille fois péri, si elle eût été réduite aux secours tardifs \& \& [sic] incertains de la froide raison“ (Roussel 1775, 48). Würde die einflussreiche weibliche Empfindsamkeit also fehlen, so die Schlussfolgerung, ,gäbe es keine Gesellschaft, keine intermediären Sozialbeziehungen. Denn dieses weibliche Gefühl bildet den ,sozialen Kitt", ohne den alles auseinanderbrechen würde" (Honegger 1992, 150).

Der „Kampf um die öffentliche Tugend“ (Pečar/Tricoire 2015, 171) spielt bei der Schaffung eines bürgerlichen Geschlechterverständnisses - und dies wird sich auch in den Moralischen Wochenschriften zeigen - eine zentrale Rolle, denn die ,philosophes', wie auch viele der Wochenschriftenproduzent*innen, ,identifizier[...] ]en sich mit den großen Rednern der Antike und trachte[...]n danach, an Stelle der Priester das Amt der Moralwächter zu übernehmen“" (ibid.).

\subsection{ZUSAMMENFASSUNG}

Die gewichtigen Umdeutungen hinsichtlich des Geschlechterverständnisses und der binären Geschlechterkonzeptionen schlagen sich im Verlauf des 18. Jahrhunderts allmählich in allen Wissens- und Lebensbereichen (wie im Verständnis von Liebe, Ehe, Leidenschaft, Erziehung) nieder. Da das neue Geschlechterwissen und mit ihm die gesellschaftlichen Auswirkungen sukzessive hervorgebracht werden - und lange Zeit auch noch parallel zueinander existieren ${ }^{29}$-, braucht es breitenwirksame Medien, wie die Moralischen Wochenschriften, mit denen die sich verändernden Rollenkonzeptionen von Frau und Mann gemeinsam mit den sich verändernden Werten, Normen und Praktiken peu à peu unter das sich konstituierende Bürgertum gebracht werden. Dieses muss nämlich erst damit vertraut gemacht werden, was es heißt, in einer Welt, die sich von den alle Lebensbereiche berührenden Dogmen der Kirche mehr und mehr löst, eine für die Gesellschaft nützliche oder unnütze Frau beziehungsweise ein nützlicher oder unnützer Mann zu sein, wobei als nützlich die Tugenden und als nutzlos die Laster erachtet werden. ${ }^{30}$

Dass dieser Paradigmenwechsel ein Prozess des Aushandelns ist, zeigt sich nicht nur anhand der zahlreichen Querelles-Texte, sondern auch in den Moralischen

29 Die binäre Geschlechterordnung setzt sich im späten 18. Jahrhundert in Form des ,ZweiGeschlechter-Modells، (Laqueur 2003) durch, womit das Geschlecht schließlich als Kategorie definiert wird. Einige Wissenschaftler*innen wie Katharina Park, Valerie Traub oder Brigitta Wrede setzen den „Prozess der medizinischen Vereindeutigung von Geschlecht bereits im 16. Jahrhundert im Zuge der (Wieder-)Entdeckung der Klitoris an“ (Klapeer 2014, 78; cf. ibid., 78-80).

30 So zum Beispiel wird in den deutschsprachigen Moralischen Wochenschriften laut Martens (1968) ganz explizit die weltliche Morallehre von der christlichen Morallehre abgegrenzt. Wiederholt wird darauf hingewiesen, dass sie einerseits ihr „Unternehmen als Ergänzung christlicher Lebenslehre, als Unterstützung der Absichten der Religion mit vernünftigen Mitteln“ (176) betrachten und dass sie andererseits „nur am diesseitigen, gesellschaftlichen Leben der Menschen, an ihrer Tugend und an ihrem Wohlergehen interessiert [sind]“ (175). 